\title{
Disturbed flow in an aquatic environment may create a sensory refuge for aggregated prey
}

\author{
Asa Johannesen ${ }^{\text {Corresp., }}{ }^{1,2}$, Alison M Dunn ${ }^{2}$, Lesley J Morrell ${ }^{3}$ \\ ${ }^{1}$ Nesvik Marine Centre, Fiskaaling, Hvalvik, Faroe Islands \\ 2 School of Biology, University of Leeds, Leeds, United Kingdom \\ 3 School of Environmental Sciences, University of Hull, Hull, United Kingdom \\ Corresponding Author: Asa Johannesen \\ Email address: asajoh@fiskaaling.fo
}

Predators use olfactory cues moved within water and air to locate prey. Because prey aggregations may produce more cue and be easier to detect, predation could limit aggregation size. However, disturbance in the flow may diminish the reliability of odour as a prey cue, impeding predator foraging success and efficiency. We explore how different cue concentrations (as a proxy for prey group size) affect risk to prey by fish predators in disturbed (more turbulent or mixed) and non-disturbed (less mixed) flowing water. We find that increasing odour cue concentration increases predation risk and disturbing the flow reduces predation risk. At high cue concentration fish were able to locate the cue source in both disturbed and non-disturbed flow, but at medium concentrations, predators only located the cue source more often than expected by chance in non-disturbed flow. This suggests that objects disturbing flow provide a sensory refuge allowing prey to form larger groups, but that group sizes may be limited by level of disturbance to the flow. 
1 Disturbed flow in an aquatic environment may create a sensory refuge for aggregated prey

2

8 Correspondence:

9 Nesvík Marine Centre, Fiskaaling (Aquaculture Research Station of the Faroe Islands), við Áir, 10 FO-430 Hvalvík, Faroe Islands

11

12 asajoh@fiskaaling.fo

$13+298214764$ 


\section{Abstract}

15 Predators use olfactory cues moved within water and air to locate prey. Because prey

16 aggregations may produce more cue and be easier to detect, predation could limit aggregation

17 size. However, disturbance in the flow may diminish the reliability of odour as a prey cue,

18 impeding predator foraging success and efficiency. We explore how different cue concentrations

19 (as a proxy for prey group size) affect risk to prey by fish predators in disturbed (more turbulent

20 or mixed) and non-disturbed (less mixed) flowing water. We find that increasing odour cue

21 concentration increases predation risk and disturbing the flow reduces predation risk. At high cue

22 concentration fish were able to locate the cue source in both disturbed and non-disturbed flow,

23 but at medium concentrations, predators only located the cue source more often than expected by

24 chance in non-disturbed flow. This suggests that objects disturbing flow provide a sensory refuge

25 allowing prey to form larger groups, but that group sizes may be limited by level of disturbance

26 to the flow.

Introduction

To avoid predation, animals use strategies from visual crypsis (Jackson et al., 2005) to

31 increased vigilance in groups (Krause and Ruxton, 2002). In cases where visual interactions

32 between predators and prey are limited, cues such as sound (Obrist et al., 1993) or detection of

33 electric fields (Kajiura and Holland, 2002) are used instead. Olfaction is a key sense used in prey

34 detection and location. Olfactory predators such as crustaceans (Gomez and Atema, 1996;

35 Weissburg and Zimmer-Faust, 1993), fish (Nevitt, 1991), and molluscs (Ferner and Weissburg, 
36 2005) can successfully track odour plumes from prey to their source. Animals use many sensing

37 strategies, including time differences in bilateral odour detection (Gardiner and Atema, 2010),

38 time-averaging of odour concentrations (Wilson and Weissburg, 2012), and making

39 simultaneous comparisons of odour concentration (Page et al., 2011; Vergassola et al., 2007). To

40 avoid such detection, prey may try to limit the amount of olfactory cue that they release or

41 otherwise make it difficult for predators to detect them (Ruxton, 2009).

42

43

44

45

46

47

48

49

50

51

52

53

54

55

56 57

To reduce the risk from predators that hunt using vision, prey can group together to increase the time taken for a hunting predator to locate them. This is known as the encounterdilution effect (Wrona and Dixon, 1991) and favours grouping as an anti-predator strategy in response to visual predators (Jackson et al., 2005; Riipi et al., 2001). However, if increasing group size makes prey increasingly easier for olfactory predators to find (Kunin, 1999), grouping may be counter-productive.

Larger or more numerous animals release more odour cue, eliciting a stronger reaction in the receiver (Hawkins et al., 2007; Kusch et al., 2004). When animals aggregate, the odour cues released interact, increasing the size and concentration of the odour plume (Villermaux and Duplat, 2003), which allows receivers to more readily track the plume to its source (Wilson and Weissburg, 2012). Grouping benefits prey avoiding olfactory predators in still water (Johannesen et al., 2014). However, water movement provides a directional cue to the prey, so olfactory cues are more easily taken advantage of in flowing rather than still water (Løkkeborg, 1998).

In a review of olfactory detection distance in insects, Andersson et al. (2013) indicate that the increase in detection with increasing size of the source is likely to be asymptotic, although theoretical work indicates accelerating detectability may also be possible (Treisman, 1975). If the 
58 risk of predation increases too much with group size, aggregation would be counterproductive in

59 species that cannot otherwise defend themselves. Here, we explore this question from the

60 perspective of three-spine sticklebacks (Gasterosteus aculeatus) locating odour sources of

61 differing concentration (as a proxy for prey group size (Hill and Weissburg, 2014; Schneider et

62 al., 2014) - but see discussion) in flowing water, to test the hypothesis that increasing prey

63 (bloodworm) cue concentration increases the risk to prey in flowing water.

64 Chemical cues are often detected in pulses because currents, turbulence, and other types

65 of disturbed flow create patches of cue (Finelli and Pentcheff, 1999; Weissburg and Zimmer-

66 Faust, 1993; Zimmer-Faust et al., 1995), which may create 'sensory refuges' (Weissburg and

67 Zimmer-Faust, 1993). When prey are in these refuges, predators may be less- or unable to detect

68 them (Ferner and Weissburg, 2005), while prey may still be able to detect predators as back

69 eddies carry odour cues 'upstream’ (Dahl et al., 1998). Prey animals occupying a sensory refuge

70 would benefit from the reduced predation success, leading to aggregation of prey in refuge areas.

71 If animals aggregate in sensory refuges, the sensory refuge may counteract the increased risk of

72 detection due to larger group size. We repeat our experiment in disturbed flow to examine the

73 additional hypothesis that disturbed flow reduces the risk to prey relative to undisturbed flow, as

74 it creates sensory refuges (Weissburg and Zimmer-Faust, 1993). Our aim is to provide an initial

75 exploration of the possible impact of prey aggregation and flow conditions on the detection of

76 prey by a foraging fish.

\section{$77 \quad$ Methods}

78 Experimental species, transportation and housing 
Two hundred three-spine sticklebacks Gasterosteus aculeatus (4-5cm total body length)

80

81

82

83

84

85

86

87

88

were caught in a pond in Saltfleet, Lincolnshire in November, $2011\left(53^{\circ} 25^{\prime} 59.55^{\prime} \mathrm{N}\right.$,

$\left.0^{\circ} 10^{\prime} 49.41^{\prime \prime} \mathrm{E}\right)$ and transported in fish bags (3-5 fish per litre) to aquarium facilities in Leeds (3

hour journey). Sticklebacks can detect and locate prey using non-visual cues in still water

(Johannesen et al., 2012), but also occupy flowing water environments and may be able to track

odour plumes to their source. Fish were housed in grey fibreglass tanks $(0.5 \times 0.5 \times 1.0 \mathrm{~m})$ with

gravel substrate, plastic plants, plant pots and mechanical filters. Light regime was 10:14 hours

light:dark, temperature was $14 \pm 2^{\circ} \mathrm{C}$ and fish were fed daily on defrosted frozen bloodworm.

Fish were kept for six months to one year for experimentation prior to release where caught in agreement with the Home Office and Defra.

\section{Procedure}

Trials were carried out in a flow-through rectangular tank $(40 \mathrm{~cm}$ by $53 \mathrm{~cm}$, water depth $9 \mathrm{~cm}$, flow velocity $3 \mathrm{~cm} \mathrm{~s}^{-1}$ ) based on a Y maze design (Ward et al., 2011) (Fig 1). The stem of the maze was $33 \mathrm{~cm}$ in length including a $20 \mathrm{~cm}$ 'release zone' with a removable barrier. Each 'arm' was $20 \mathrm{~cm}$ long. Conditioned water was pumped from a header tank into the maze, entered the maze over a horizontal barrier in both arms of the $\mathrm{Y}$, and passed through a collimator to create an even water flow ("undisturbed flow"). Flow characteristics were not measured, but pilot trials using dye indicated that the odour cue would move through the water evenly. Water left the flume through 3 mesh-covered exit holes evenly spaced across the base of the stem of the Y, and was not re-circulated. Trials were observed from behind a screen via a webcam to reduce disturbance to the fish. 
102 low: $5 \mathrm{~g} \mathrm{l}^{-1}$ medium: $10 \mathrm{~g} \mathrm{l}^{-1}$ and high: $20 \mathrm{~g} \mathrm{l}^{-1}$ ). Although spatial distribution of aggregated prey

103 affects odour plume composition, we use prey cue concentration as a proxy for size or number of

104 prey. This is because the interacting odour plumes from multiple prey individuals will increase

105 cue concentration (Hawkins et al., 2007; Villermaux and Duplat, 2003) and while adding the

106 spatial factor to this experiment would be more realistic, it would also complicate the

107 experiment. Cues were delivered to the maze using two peristaltic pumps at a rate of $10 \mathrm{ml} \mathrm{min}^{-1}$

108 (source diameter $4 \mathrm{~mm}$, velocity $1.3 \mathrm{~cm} \mathrm{~s}^{-1}$ ). In each trial, the olfactory cue entered at one arm of

109 the maze, and a conditioned water control (containing red food dye to copy the tint of the

110 bloodworm cue water) entered at the other at the same rate. Cue side was allocated at random to

111 control for side preference. After the trial, the maze was emptied and refilled with conditioned

112 water to remove olfactory cues from the previous trial.

114 until they resumed normal behaviour (start - stop swimming at moderate speed, five minutes

115 minimum). Following acclimatisation, the water inlet pump was switched on and ran for two

116 minutes (to stabilise flow) before cue pumps were turned on. The behaviour of the test fish was

117 monitored until it had visited both sides of the stem of the Y (two minutes minimum) and the

118 barrier was raised using a pulley system. The fish was allowed five minutes to reach the top of

119 one arm of the Y, where its choice (cue or control) was recorded. The time taken for the fish to

120 acclimatise (begin swimming) and the time taken to reach the top of the chosen arm were also

121 recorded. Fish were excluded from the experiment if they did not resume normal behaviour in

122 the release zone $(\mathrm{N}=23)$, did not visit both sides of the stem of the $\mathrm{Y}$ within 5 minutes $(\mathrm{N}=8$ 
123 fish) or did not make a choice $(\mathrm{N}=6)$. Final sample sizes in undisturbed flow were: low: $\mathrm{N}=16$,

124 medium: $\mathrm{N}=16$, high: $\mathrm{N}=16$ ).

125

We subsequently investigated the effect of disturbed flow on stickleback choice in the

126 maze. Three cylinders were placed in each arm of the Y maze to create downstream disruption to

127 the flow (see Fig 1). Visualisation of the flow using food dye indicated that the cylinders caused

128 the odour plumes to split and disperse, and that the plumes appeared qualitatively different to

129 those in the experiment with no disturbance to the flow. Methods were the same as in the

130 previous experiment, but investigated only two cue concentrations: medium and high. The low

131 concentration was not used as the first experiment indicated that fish did not show a preference at

132 this concentration (see results). Eight fish were excluded from this experiment, giving final

133 sample sizes of $\mathrm{N}=17$ for medium cue concentration and $\mathrm{N}=17$ for high cue concentration in

134 disturbed flow. Each fish was used only once in the experiments, and different fish were used in

135 the two flow conditions to avoid any learning effects. The total sample size for both experiments

136 was $\mathrm{N}=82$.

137 Analysis

Data were analysed using R v 2.13.0 (R Core Team, 2013). Cox proportional hazards

139 survival models were used to analyse fish time to acclimatise and time to choose (survival

140 package in R; (Therneau and Lumley, 2011)). Survival models are highly flexible and useful for

141 time-to type data, especially when data do not follow a Gaussian distribution and contain

142 censored times (Therneau and Grambsch, 2000). Preference for prey cue was tested using

143 binomial exact tests against an expectation of random (0.5). A correction for false discovery rate

144 was performed (Benjamini and Hochberg, 1995) on the tests to control for multiple comparisons 
145 and the adjusted $\mathrm{p}$ values are given in the results section. A binomial GLM with choice as

146 dependent variable (cue, no cue) and cue concentration and flow type as independent variables

147 was used to test the effect of treatments on choice.

148

149

Ethical statement

150

As experiments with fish fall outside of the remit of the University of Leeds Ethical

151

Board and no licensed procedures were used, this study was not subject to ethical review.

152

However, laboratory experiments were carried out in accordance with University of

153 Leeds guidelines and in agreement with Home Office licensed technical staff at the animal

154 facility. Great care was taken to ensure optimal welfare for all fish involved in this study.

155

156 Results

157

Fish tested in the disturbed flow condition took less time to acclimatise than those in the 158 non-disturbed flow condition (coxph: Chi-squared $=25.81, \mathrm{df}=1, \mathrm{p}<0.001$ ), but there was no

159 effect of cue concentration or flow type on time to choose once acclimatised (coxph: Chi-squared $160=6.22, \mathrm{df}=5, \mathrm{p}=0.29$.

161 In the 'undisturbed flow' condition, fish selected the cue arm over the control arm at 162 medium $(\mathrm{N}=13 / 16, \mathrm{P}($ success $)=0.8125, \mathrm{p}=0.035)$ and high $(\mathrm{N}=15 / 16, \mathrm{P}($ success $)=0.938, \mathrm{p}$ $163=0.003)$ cue concentrations, but not at the low cue concentration $(\mathrm{N}=11 / 16, \mathrm{P}($ success $)=0.688$, $164 \mathrm{p}=0.26)$. When flow disturbance was added, fish preferentially selected the cue arm at high ( $\mathrm{N}$ 
$165=14 / 17, \mathrm{P}($ success $)=0.824, \mathrm{p}=0.033)$ but not medium $(\mathrm{N}=10 / 17, \mathrm{P}($ success $)=0.588, \mathrm{p}=$

166 0.629) cue concentrations (Fig 2).

167 In a test of the effects of cue concentration and flow regime, cue concentration

168 significantly affected choice (Binomial GLM; $z=2.235, \mathrm{~N}=82, \mathrm{p}=0.025$ ) while the effect of

169 water regime fell short of significance (Binomial GLM; $\mathrm{z}=-1.814, \mathrm{~N}=82, \mathrm{p}=0.070$ ).

170

171 Discussion

172 Our results suggest that in a Y-maze with olfactory cue presented in one arm only, fish

173 predators can successfully choose the arm containing the cue more often than expected by

174 chance if the concentration of the cue is high enough. At the low cue concentration, fish did not

175 choose the cue arm more often than the non-cue arm. Interpreted in the context of our question of

176 how increasing group size affects detection, this suggests that grouping in prey (increased cue

177 concentration) may increase risk from olfactory predators. Adding objects to the maze to disturb

178 the flow (i.e. create more turbulent mixing) decreased the number of successful choices,

179 particularly at the medium cue concentration, suggesting that 'sensory refuges' created by

180 disturbed flow (Weissburg and Zimmer-Faust, 1993) allow larger groups to form by countering

181 the increased risk of detection. However, at higher cue concentrations, the benefit gained from

182 flow disturbance may decrease, and larger groups would be more easily detected. While we did

183 not compare multiple disturbance levels, we suggest that the level of disturbance may also

184 influence the detection of prey cues of different concentrations.

185 We used concentration as a proxy for group size, following previous work (Schneider et $186 a l ., 2014)$, although the effect of group size on odour plumes is more complex. When individuals 
187 group together, they produce a greater number of odour filaments (Monismith et al., 1990;

188 Wilson and Weissburg, 2012) that cover a wider area (Webster and Weissburg, 2001). When

189 prey are spaced sufficiently far apart, individual odour filaments do not interact and would not

190 result in an increase in cue concentration. In that case, the risk of detection by predators who are

191 unable to detect any individual filament, but would detect a more concentrated one, remains low

192 regardless of group size. However, in cases of tightly aggregated prey, filaments are likely to

193 interact and increase the time-averaged concentration (Villermaux and Duplat, 2003) used by

194 some predators to track plumes (Ferner and Weissburg, 2005) as well as filament concentration

195 (Villermaux and Duplat, 2003). Therefore, tightly packed prey increase risk of detection with

196 group size, particularly by predators who would not have detected a single individual's odour

197 plume.

198

Concentration may reflect a number of other features of the prey landscape in addition to

199 group size in tight aggregations. As cues become more diluted over distance and time, cue

200 concentration may signal distance to prey (but see Bytheway et al., 2013), or if larger individuals

201 release more cue, the concentration could signal size of prey. Thus, predators could move

202 towards higher concentrations because they represent better value predation opportunities, rather

203 than because they are easier to detect. However, while the results in this experiment could reflect

204 a perceived value of cue in the fish rather than simple detection, there is no indication of this in

205 the latency to choose a cue arm in our data.

206 While turbulence or other disturbance to flow can cause odour plumes to break up

207 (Webster and Weissburg, 2001) it can also act to mix the plumes and dilute the cue to

208 background levels with only intermittent spikes (Webster and Weissburg, 2009) that may not be

209 worth exploring. Either mechanism would act to make tracking the cue to the source more 
210 difficult for the predator (Robinson et al., 2011), although this may depend on the predator's

211 sensing strategy and sensitivity (Ferner and Weissburg, 2005). Our observations with food dye

212 suggest that the plume in disturbed flow split primarily into two meandering plumes. Assuming a

213 fish was only exposed to one arm of the split plume, the decreased amount of cue could mean

214 fewer or smaller prey, greater distance to prey, or the concentration might be below a detection

215 threshold. A meandering plume will, in addition to the perceived lower reward, be more difficult

216 to track for fish and other filament samplers, making the effort greater. While a time-averaging

217 predator may be able to compensate for meandering plumes (Page et al., 2011), a diluted and

218 split plume signals lower reward, so inhabiting turbulent water can also benefit tightly packed

219 prey hiding from a time-averager. However, as sticklebacks most likely do not use time-

220 averaging sensing strategies (Nevitt, 1991; Webster and Weissburg, 2001), this has not been

221 explicitly tested here.

Our study did not investigate the fluid mechanics and transport of olfactory cue in the

223 different flow regimes, focusing instead on the response of the predator. Thus, we cannot

224 speculate on the sensory mechanism, motivation, or features of water flow and cue transport that

225 cause the different behaviours shown by our fish predators. However, the end result for the prey

226 remains the same. If an olfactory prey cue is highly concentrated, indicating either great reward

227 (many or large prey) or easy reward (close proximity) a predator is more likely to pursue that

228 cue. Conversely, if the olfactory prey cue plume is somehow broken down, indicating small

229 reward (few or small prey) or difficult reward (long distance, a plume that is difficult to track) a

230 fish predator is less likely to pursue that cue. In the context of our question regarding

231 aggregation, this suggests prey are able to aggregate into larger groups by taking advantage of a

232 sensory refuge and either fooling the predator into thinking they are not worth the effort (small 
233 reward/high effort) or decreasing the cue to avoid detection. Individuals in such aggregations

234 would in turn benefit from greater survival chance if found due to other benefits of grouping, 235 such as predator satiation.

The study of anti-predator aggregation has primarily focused on predators that use vision

237 to detect their prey (Ioannou and Krause, 2008), while the effect of olfactory predators on the

238 evolution of aggregation is less well understood. Our work suggests that group size may interact

239 with environmental parameters, and that the evolution of grouping in response to olfactory

240 predators may be dependent on the flow environment. However, further work is needed to fully

241 investigate the relationship between grouping prey, detection by predators, and environmental

242 conditions. Prey are known to aggregate in streams (Rasmussen and Downing, 1988), but

243 aggregation decisions may depend on factors other than risk from olfactory predators, including

244 foraging opportunities, flow speed and risk from predators relying on other sensory modalities

245 (Ioannou and Krause, 2008; Krause and Ruxton, 2002). Experimental manipulation and

246 characterisation of flow regimes and the response of predators and prey may help disentangle the

247 interacting effects of group size, flow regime and aggregation in response to other resources.

\section{Data Accessibility Section}

251 (http://dx.doi.org/10.6084/m9.figshare.985515).

\section{References}

253 Andersson, P., Löfstedt, C. and Hambäck, P. A. (2013) How insects sense olfactory patches - the 254 spatial scaling of olfactory information, Oikos, 122 (7), pp. 1009-1016. DOI:10.1111/j.1600- 
256 Benjamini, Y. and Hochberg, Y. (1995) Benjamini Y, Hochberg Y. Controlling the false 257 discovery rate: a practical and powerful approach to multiple testing, Journal of the Royal 258 Statistical Society B, 57 (1), pp. 289-300. DOI:10.2307/2346101.

259 Bytheway, J. P., Carthey, A. J. R. and Banks, P. B. (2013) Risk vs. reward: how predators and 260 prey respond to aging olfactory cues, Behavioral Ecology and Sociobiology, 67 (5), pp. 715-725. 261 DOI:10.1007/s00265-013-1494-9.

262 Dahl, J., Anders Nilsson, P. and Pettersson, L. B. (1998) Against the flow: chemical detection of 263 downstream predators in running waters, Proceedings of the Royal Society B: Biological 264 Sciences, 265 (1403), pp. 1339-1344. DOI:10.1098/rspb.1998.0439.

265 Ferner, M. C. and Weissburg, M. J. (2005) Slow-moving predatory gastropods track prey odors 266 in fast and turbulent flow., The Journal of Experimental Biology, 208 (5), pp. 809-819.

267 DOI:10.1242/jeb.01438.

268 Finelli, C. M. and Pentcheff, N. (1999) Odor transport in turbulent flows: constraints on animal 269 navigation, Limnology and ..., 44 (4), pp. 1056-1071.

270 Gardiner, J. M. and Atema, J. (2010) The function of bilateral odor arrival time differences in 271 olfactory orientation of sharks., Current Biology : CB, 20 (13), pp. 1187-91.

272 DOI:10.1016/j.cub.2010.04.053.

273 Gomez, G. and Atema, J. (1996) Temporal resolution in olfaction: stimulus integration time of 274 lobster chemoreceptor cells, The Journal of Experimental Biology, 199 (Pt 8), pp. 1771-9.

275 Hawkins, L., Magurran, a and Armstrong, J. (2007) Innate abilities to distinguish between 276 predator species and cue concentration in Atlantic salmon, Animal Behaviour, 73 (6), pp. 1051277 1057. DOI:10.1016/j.anbehav.2006.08.011.

278 Hill, J. M. and Weissburg, M. J. (2014) Crabs interpret the threat of predator body size and 279 biomass via cue concentration and diet, Animal Behaviour, 92, pp. 117-123.

280 DOI:10.1016/j.anbehav.2014.03.025.

281 Ioannou, C. C. and Krause, J. (2008) Searching for prey: the effects of group size and number, 282 Animal Behaviour, 75 (4), pp. 1383-1388.

283 Jackson, A. L., Brown, S., Sherratt, T. N. and Ruxton, G. D. (2005) The effects of group size, 284 shape and composition on ease of detection of cryptic prey, Behaviour, 142 (6), pp. 811-826. 285 DOI:10.1163/1568539054729105.

286

287

288

289

290

291

292

293
Johannesen, A., Dunn, A. M. and Morrell, L. J. (2012) Olfactory cue use by three-spined sticklebacks foraging in turbid water: prey detection or prey location?, Animal Behaviour, 84 (1), pp. 151-158. DOI:10.1016/j.anbehav.2012.04.024.

Johannesen, A., Dunn, A. M. and Morrell, L. J. (2014) Prey aggregation is an effective olfactory predator avoidance strategy, PeerJ, 2 (May), pp. e408. DOI:10.7717/peerj.408.

Kajiura, S. M. and Holland, K. N. (2002) Electroreception in juvenile scalloped hammerhead and sandbar sharks, The Journal of Experimental Biology, 205 (23), pp. 3609-3621.

Krause, J. and Ruxton, G. D. (2002) Living in Groups. Vol. I. Oxford University Press. 
294 Kunin, W. E. (1999) Patterns of herbivore incidence on experimental arrays and field

295 populations of ragwort, Senecio jacobaea, Oikos, 84 (3), pp. 515-525.

296 Kusch, R. C., Mirza, R. S. and Chivers, D. P. (2004) Making sense of predator scents:

297 investigating the sophistication of predator assessment abilities of fathead minnows, Behavioral

298 Ecology and Sociobiology, 55 (6), pp. 551-555. DOI:10.1007/s00265-003-0743-8.

299 Løkkeborg, S. (1998) Feeding behaviour of cod, Gadus morhua: activity rhythm and chemically 300 mediated food search, Animal Behaviour, 56 (2), pp. 371-378. DOI:10.1006/anbe.1998.0772.

301 Monismith, S. G., Koseff, J. R., Thompson, J. K., O’Riordan, C. A. and Nepf, H. M. (1990) A

302 study of model bivalve siphonal currents., Limnology and Oceanography, 35 (3), pp. 680-696.

303 Nevitt, G. A. (1991) Do fish sniff? A new mechanism of olfactory sampling in pleuronectid

304 flounders., The Journal of Experimental Biology, 157, pp. 1-18.

305 Obrist, M. K., Fenton, M. B., Eger, J. L. and Schlegel, P. a (1993) What ears do for bats: a

306 comparative study of pinna sound pressure transformation in chiroptera., The Journal of

307 Experimental Biology, 180, pp. 119-52.

308 Page, J. L., Dickman, B. D., Webster, D. R. and Weissburg, M. J. (2011) Staying the course:

309 chemical signal spatial properties and concentration mediate cross-stream motion in turbulent

310 plumes., The Journal of Experimental Biology, 214 (Pt 9), pp. 1513-1522.

311 DOI:10.1242/jeb.049304.

312 R Core Team (2013) R: A language and environment for statistical computing. Vienna, Austria:

313 R Foundation for Statistical Computing.

314 Rasmussen, J. B. and Downing, J. A. (1988) The spatial response of chironomid larvae to the 315 predatory leech Nephelopsis obscura, The American Naturalist, 131 (1), pp. 14-21.

316 DOI:10.1086/284770.

317 Riipi, M., Alatalo, R. V, Lindström, L. and Mappes, J. (2001) Multiple benefits of 318 gregariousness cover detectability costs in aposematic aggregations., Nature, 413 (6855), pp. 319 512-4. DOI:10.1038/35097061.

320 Robinson, E. M., Smee, D. L. and Trussell, G. C. (2011) Green crab (Carcinus maenas) foraging 321 efficiency reduced by fast flows., PloS One, 6 (6), pp. e21025.

322 DOI:10.1371/journal.pone.0021025.

323 Ruxton, G. D. (2009) Non-visual crypsis: a review of the empirical evidence for camouflage to 324 senses other than vision., Philosophical Transactions of the Royal Society of London. Series B,

325 Biological Sciences, 364 (1516), pp. 549-57. DOI:10.1098/rstb.2008.0228.

326 Schneider, J., Worischka, S., Hellmann, C., Benndorf, J. and Winkelmann, C. (2014) Flexibility

327 in feeding periodicity of a grazing mayfly in response to different concentrations of benthivorous

328 fish, Limnologica - Ecology and Management of Inland Waters, 45, pp. 24-32.

329 DOI:10.1016/j.limno.2013.10.002.

330 Therneau, T. M. and Grambsch, P. M. (2000) Modeling Survival Data: Extending the Cox 331 Model. London: Springer.

332 Therneau, T. M. and Lumley, T. (2011) survival: Survival analysis, including penalised 
333 likelihood: $\mathrm{R}$ package version 2.36-5.

334 Treisman, M. (1975) Predation and the evolution of gregariousness. I. Models for concealment

335 and evasion, Animal Behaviour, 23, pp. 779-800. DOI:10.1016/0003-3472(75)90106-2.

336 Vergassola, M., Villermaux, E. and Shraiman, B. I. (2007) 'Infotaxis' as a strategy for searching

337 without gradients., Nature, 445 (7126), pp. 406-9. DOI:10.1038/nature05464.

338 Villermaux, E. and Duplat, J. (2003) Mixing is an aggregation process, Comptes Rendus

339 Mécanique, 331 (7), pp. 515-523. DOI:10.1016/S1631-0721(03)00110-4.

340 Ward, A. J. W., Herbert-Read, J. E. and Simpson, S. J. (2011) Diets and decisions: the potential 341 use of food protein cues in dietary, sexual and social decisions by mosquitofish, Animal

342 Behaviour, 82 (4), pp. 783-790. DOI:10.1016/j.anbehav.2011.07.010.

343 Webster, D. R. and Weissburg, M. J. (2001) Chemosensory guidance cues in a turbulent

344 chemical odor plume, Limnology and Oceanography, 46 (5), pp. 1034-1047.

345 Webster, D. R. and Weissburg, M. J. (2009) The Hydrodynamics of Chemical Cues Among

346 Aquatic Organisms, Annual Review of Fluid Mechanics, 41 (1), pp. 73-90.

347 DOI:10.1146/annurev.fluid.010908.165240.

348 Weissburg, M. J. and Zimmer-Faust, R. K. (1993) Life and death in moving fluids:

349 hydrodynamic effects on chemosensory-mediated predation, Ecology, 74 (5), pp. 1428.

350 DOI:10.2307/1940072.

351

352

353

354

355

356

357

358

359

360
Wilson, M. L. and Weissburg, M. J. (2012) Temporal and spatial sampling strategies maintain tracking success of whelks to prey patches of differing distributions, Animal Behaviour, 84 (6), pp. 1323-1330. DOI:10.1016/j.anbehav.2012.08.024.

Wrona, F. J. and Dixon, R. W. J. (1991) Group size and predation risk: a field analysis of encounter and dilution effects, The American Naturalist, 137 (2), pp. 186-201. DOI:10.1086/285153.

Zimmer-Faust, R. K., Finelli, C. M., Pentcheff, N. D. and Wethey, D. S. (1995) Odor plumes and animal navigation in turbulent water flow: a field study, Biological Bulletin, 188 (2), pp. 111116. DOI:10.2307/1542075. 


\section{Figure 1 (on next page)}

Layout of the Y-maze (Total dimensions: length $93 \mathrm{~cm}$ and width $40 \mathrm{~cm}$ ).

Water flowed over the horizontal barrier (mid-way in the $30 \mathrm{~cm}$ long header chamber) before entering the Y-maze through a collimator (10 cm long) to ensure even flow on both sides. The water flowed along the arms of the $Y(20 \mathrm{~cm}$ long) before entering the stem (33 cm long), which was partitioned with a removable barrier for the release zone $(20 \mathrm{~cm}$ long). Water flowed through the $Y$ maze at approximately $3 \mathrm{~cm} \mathrm{~s}-1$ before exiting through the outlet holes ( $3 \mathrm{~cm}$ in diameter). Cue input points are marked by a black dot. Large open circles represent the cylinders $(5.5 \mathrm{~cm}$ in diameter) added to the tank in the 'disturbed flow' treatments. 


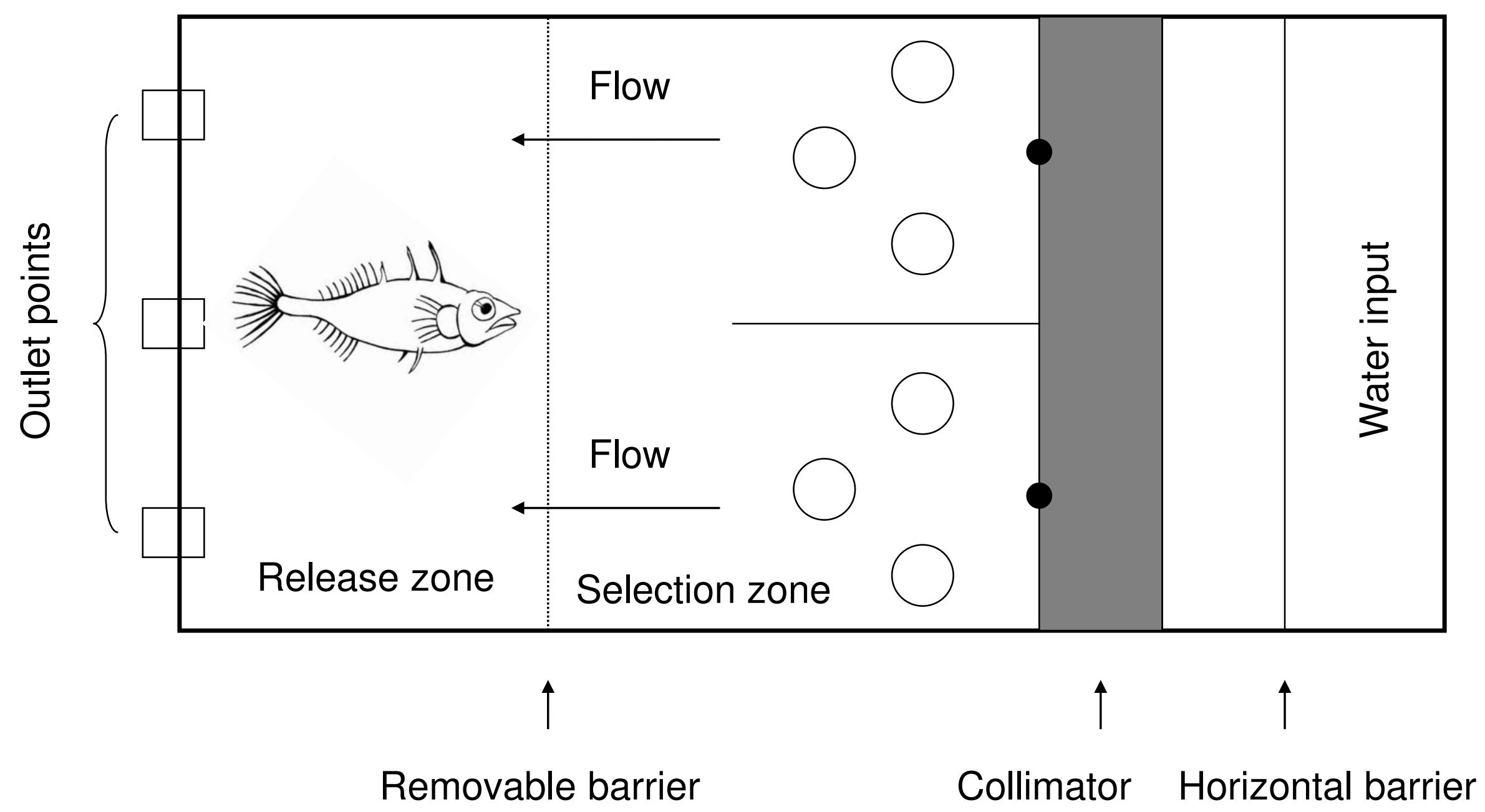




\section{Figure 2 (on next page)}

Proportion of fish choosing the prey side in a y-maze.

Stars above bars signify significant differences (binomial exact tests) from random choice of side. Dark bars are non-disturbed flow treatments and light bars are disturbed flow treatments. The horizontal dashed line indicates no preference. 


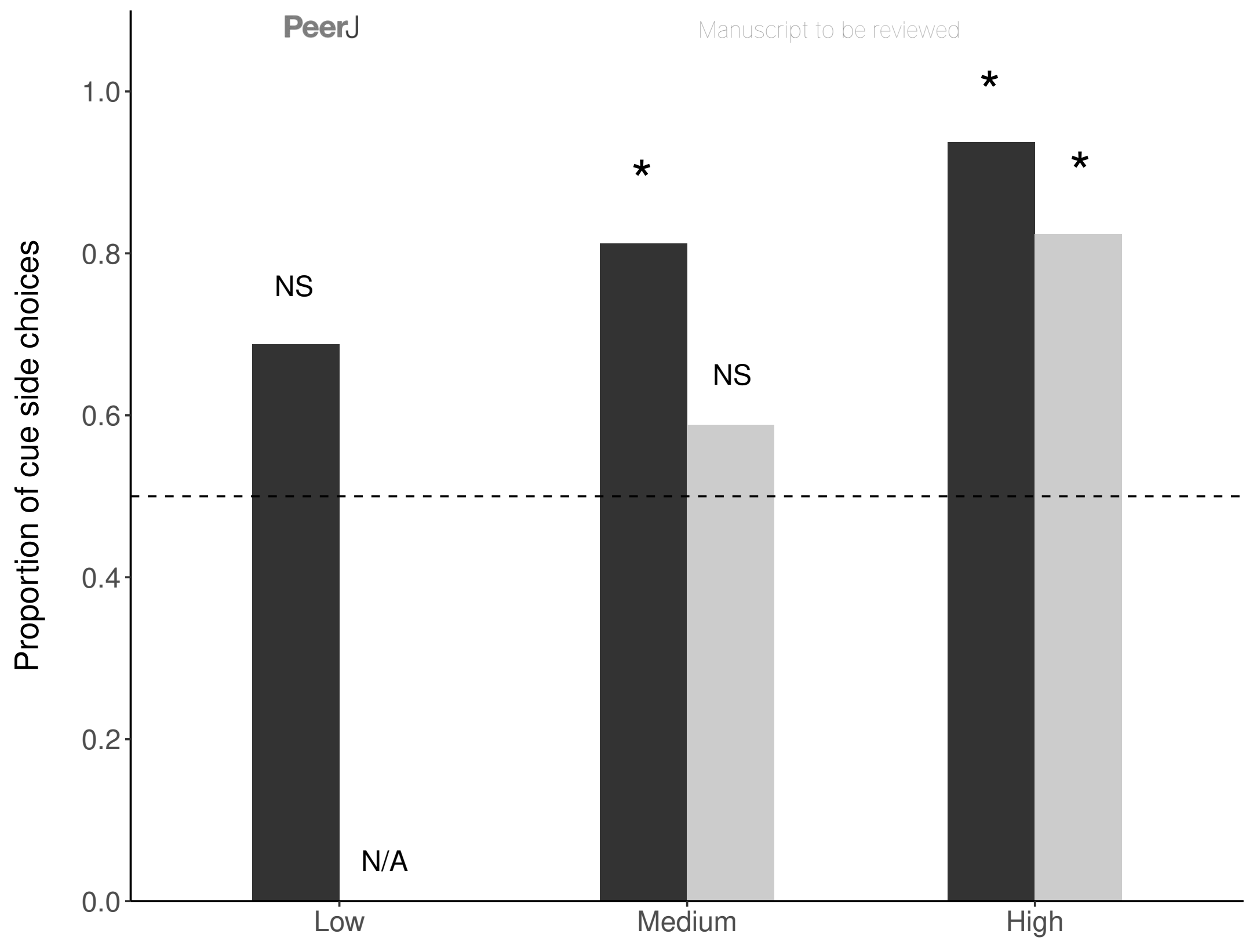

